\title{
Supercapacitors Based on c-Type Cytochromes Using Conductive Nanostructured Networks of Living Bacteria
}

\author{
Nikhil S. Malvankar, ${ }^{*[a, b]}$ Tünde Mester, $^{[b]}$ Mark T. Tuominen, $^{[a]}$ and Derek R. Lovley ${ }^{[b]}$
}

\begin{abstract}
Supercapacitors have attracted interest in energy storage because they have the potential to complement or replace batteries. Here, we report that c-type cytochromes, naturally immersed in a living, electrically conductive microbial biofilm, greatly enhance the device capacitance by over two orders of magnitude. We employ genetic engineering, protein unfolding and Nernstian modeling for in vivo demonstration of charge storage capacity of c-type cytochromes and perform electrochemical impedance spectroscopy, cyclic voltammetry and
\end{abstract}

charge-discharge cycling to confirm the pseudocapacitive, redox nature of biofilm capacitance. The biofilms also show low self-discharge and good charge/discharge reversibility. The superior electrochemical performance of the biofilm is related to its high abundance of cytochromes, providing large electron storage capacity, its nanostructured network with metallic-like conductivity, and its porous architecture with hydrous nature, offering prospects for future low cost and environmentally sustainable energy storage devices.

\section{Introduction}

Supercapacitors have emerged as novel energy storage devices to complement or replace batteries in high-power applications such as electric devices and vehicles, ${ }^{[1,2]}$ but improvements in the amount of capacitance or energy stored are necessary for most practical applications. ${ }^{[3]}$ There is the possibility that capacitance of redox-active materials can be combined with double-layer capacitors to maximize supercapacitance for the development of future-generation, high-energy supercapacitors. ${ }^{[3]}$ Biological materials are relatively inexpensive and environmentally sustainable and might be used to significantly enhance device performance. For example, genetically engineered viruses with peptides have been used to improve battery capacity. ${ }^{[4]}$ The viruses coat themselves with iron phosphate and then bind specifically to carbon nanotubes, forming a battery cathode in which electrons can travel from carbon nanotube to the iron phosphate in a short time. ${ }^{[4]}$

Supercapacitors are generally classified as electrical doublelayer capacitors, where the capacitance arises due to the electrostatic charge accumulation at the electrode/electrolyte interface, or as pseudocapacitors, where electro-active species cause fast surface redox reactions. ${ }^{[2]}$ The heme groups of ctype cytochromes readily interconvert between oxidation states over a very wide redox potential window, which makes c-type cytochromes highly efficient for rapid electron exchange. The microorganism Geobacter sulfurreducens, which respires by reducing metals, ${ }^{[5]}$ contains the highest number of genes coding for $c$-type cytochromes ${ }^{[6]}$ and most of them have multiple hemes. ${ }^{[6]}$ It has been proposed that these abundant multi-heme c-type cytochromes in the periplasm and the outer surface of $G$. sulfurreducens play an important ecological role, permitting temporary storage of electrons in cytochromes and thus continued, short-term electron transfer across the inner membrane when natural electron acceptors, such as Fe ${ }^{\text {III }}$ oxides, are temporarily unavailable. ${ }^{[7]}$ This is a fundamental mechanism for a broad range of biochemical and geological processes in natural environments and for the development of novel methods of energy generation. ${ }^{[8]}$ For example, it has been suggested that the capacitor behavior plays an important role for bioremediation of uranium. ${ }^{[9]}$

Although it has been suggested that the c-type cytochromes in G. sulfurreducens biofilms act as electron sinks and can also function as capacitors, ${ }^{[10,11]}$ capacitance was not measured and it had not been demonstrated that c-type cytochromes were storing electrons.

Here, we report on direct measurements of the in vivo capacitance of $G$. sulfurreducens biofilms and demonstrate that faradic reactions of the cytochromes confer large pseudocapacitance to biofilms with a specific capacitance that is comparable to synthetic supercapacitors. ${ }^{[1]}$ These findings suggest new prospects for future low-cost and environmentally sustainable energy storage devices.

[a] Dr. N. S. Malvankar, Prof. M. T. Tuominen

Department of Physics

University of Massachusetts, Amherst

203, Morrill Science Center IVN

639 North Pleasant Street, Amherst, MA 01003 (USA)

Fax: $(+1)$ 413-577-4660

E-mail:nikhil@physics.umass.edu

[b] Dr. N. S. Malvankar, Dr. T. Mester, ${ }^{+}$Prof. D. R. Lovley

Department of Microbiology

University of Massachusetts, Amherst (USA)

$\left.{ }^{+}\right]$Current address:

University of Michigan Medical School and Veterans Affairs Medical Research Center, Ann Arbor, Michigan 48105, USA.

Supporting information for this article is available on the WWW under http://dx.doi.org/10.1002/cphc.201100865. 


\section{Results and Discussion}

\subsection{Direct Measurements of Biofilm Capacitance}

In microbial fuel cells in which two gold anodes separated by a non-conducting gap were provided as electron acceptors (Figure 1), biofilms of $G$. sulfurrreducens grew across the non-

a)

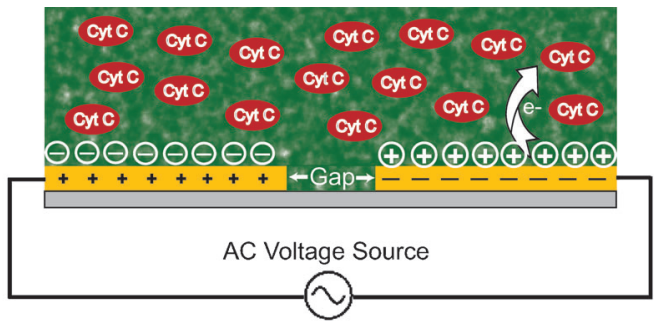

b)

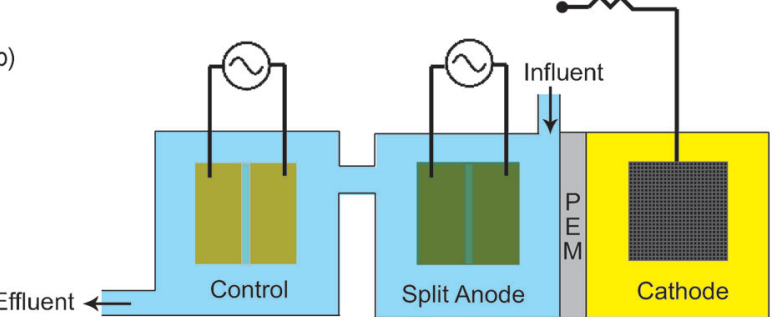

Figure 1. a) Split-electrode geometry and capacitance formation. Gold electrodes separated by a $50 \mu \mathrm{m}$ non-conductive gap are shown in yellow. Electrostatic double layer ions are shown in white and c-type cytochromes enabling pseudocapacitance are shown in red. The conductive biofilm matrix is shown in green. b) Setup for two-electrode ac impedance spectroscopy measurements. The anode and cathode of the microbial fuel cell were temporarily disconnected and an ac voltage was applied across the split-anode (configuration shown). PEM: Proton exchange membrane. Biofilm immersed in an electrolyte (blue) is shown in green.

conducting gap, as previously described. ${ }^{[12]}$ When biofilm capacitance was measured by applying ac voltage across the split electrodes (Figure $1 \mathrm{~b}$ ), the impedance data could be analyzed with the previously described ([12] and references therein) equivalent circuit model (Figure 2). As the biofilms grew over the electrodes, the capacitance increased by two orders of magnitude over the capacitance of the double-layer capacitor that formed when bare split electrodes were immersed in the medium (Figure 3).

When $G$. sulfurreducens was grown with the alternate electron acceptor fumarate, there was no increase in capacitance over time (Figure 4). Fumarate-grown biofilms exhibit low conductivity even though they bridge the non-conducting gap. ${ }^{[12]}$ In a similar manner, biofilms of Escherichia coli and Pseudomonas aeruginosa, which can also bridge the gap, but are nonconductive, ${ }^{[12]}$ did not have significant capacitance. These results demonstrated that the ability for electron conduction through the biofilm, which in G. sulfurreducens can be attributed to a network of conductive pili, ${ }^{[12]}$ is necessary for the biofilm to function as a capacitor.

Furthermore, the high porosity of the biofilms, which are comprised of $>90-95 \%$ electrolyte, ${ }^{[13]}$ provides high electrolyt-

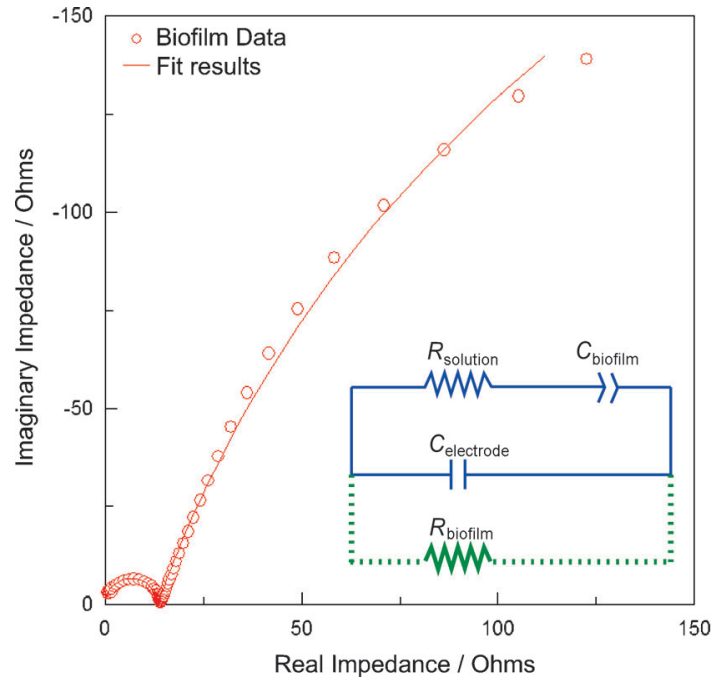

Figure 2. Representative impedance spectrum of Geobacter sulfurreducens biofilm. Red open circles show the impedance data and red line shows the fit to the impedance data using equivalent circuit model presented in the inset. Impedance spectrum for control electrodes is presented in the Supporting Information. Inset: Equivalent circuit used to extract capacitance values from the impedance spectra. $R_{\text {solution }}$ represents ionic conductivity of the electrolyte, $C_{\text {biofilm }}$ is the capacitance at the biofilm-electrode interface, $C_{\text {electrode }}$ is the geometric capacitance due to electrodes and $R_{\text {biofilm }}$ is due to electronic conductivity of the biofilm. ${ }^{[12]}$

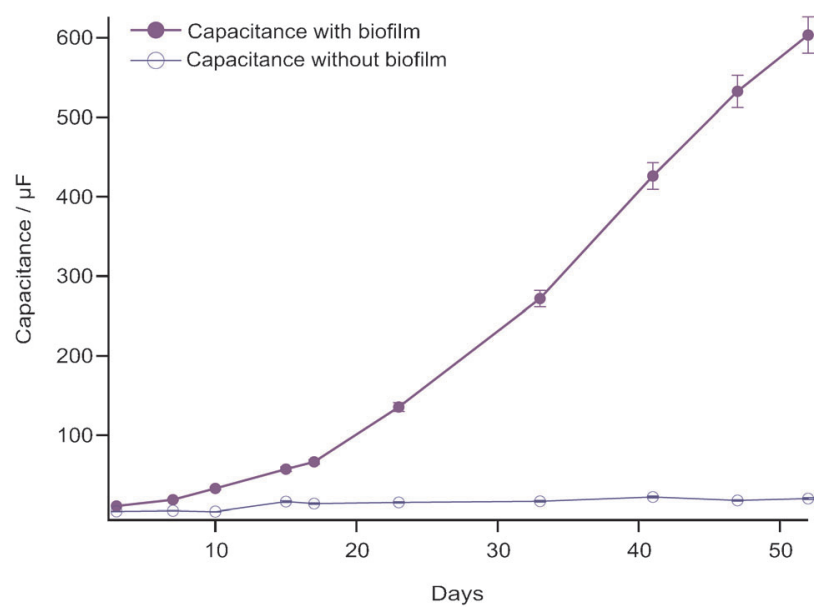

Figure 3. Capacitance increase due to the biofilm. Capacitance over days in split electrode bridged by a G. sulfurreducens biofilm (blue solid circles) and corresponding bare split-electrodes (control) without biofilm (blue open circles). Error bars: Standard deviation. Capacitance was measured with twoelectrode ac impedance spectroscopy.

ic accessibility for the movement of ions that is necessary to maintain electroneutrality associated with the capacitor formation.

As previously reported ${ }^{[12]}$ removing the electron donor acetate from the media completely suppressed the fuel cell current (Figure 5). However, there was no impact on capacitance (Figure 5). When acetate additions were resumed, current production returned to its original rate. The finding that a biofilm of metabolically inactive cells had the same capacitance as a 


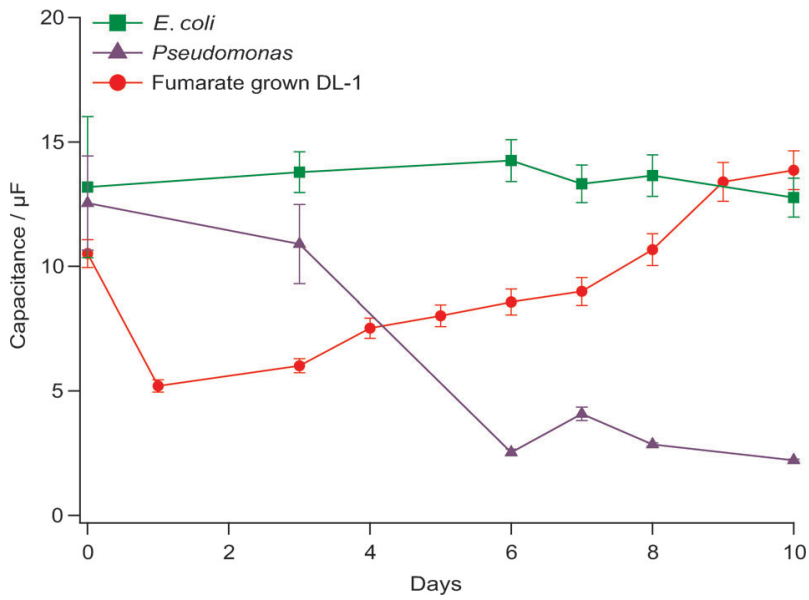

Figure 4. Capacitance over days in split electrode bridged by non-conductive biofilms. Green: Escherichia coli biofilm, Violet: Pseudomonas aeruginosa biofilm, Red: G. sulfurreducens biofilm grown with fumarate as electron acceptor. Error bars: Standard deviation. Capacitance was measured with twoelectrode ac impedance spectroscopy.

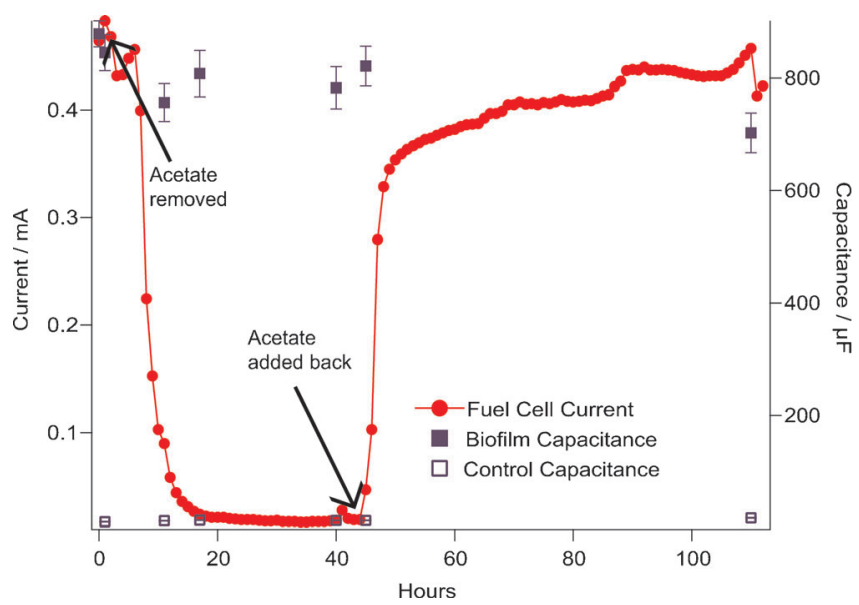

Figure 5. Effect of acetate removal on Geobacter sulfurreducens fuel cell current and biofilm capacitance. Error bars: Standard deviation. Capacitance was measured with two-electrode ac impedance spectroscopy.

current-producing biofilm suggested that the capacitance was associated with a stable component of the biofilm, rather than a transient molecule that required continuous replenishment through metabolic activity. The fact that $G$. sulfurreducens biofilms exhibit metallic-like conductivity, ${ }_{1}^{[12]}$ providing efficient pathways between the electrode and electro-active species in the biofilm, suggests that the charge transfer causing the capacitance is due to the electrons present within the biofilm.

\subsection{Contribution of Cytochromes to Biofilm Capacitance}

It was previously proposed that c-type cytochromes confer capacitance to individual cells of $G$. sulfurreducens. ${ }^{[7]}$ To evaluate whether the capacitance of biofilms could be attributed to $c$ type cytochromes, the capacitance of the biofilms of different strains of $G$. sulfurreducens with different capabilities for cyto- chrome production was evaluated. For example, the c-type cytochromes OmcB, OmcE, OmcS, and OmcT are among the most abundant outer surface cytochromes of $G$. sulfurreducens. ${ }^{[14]}$ In strain ST, the genes for OmcS and OmcT have been deleted ${ }_{1}^{[15]}$ whereas in strain BEST, the genes for OmcB and OmcE have also been deleted. ${ }^{[14]}$ The biofilms of both of these strains had a lower content of cytochrome hemes and less capacitance than the wild-type DL-1 strain (Figure 6). Strain KN400, which produces higher current densities than strain DL-1 but has a lower cytochrome content, ${ }^{[16]}$ had a biofilm heme content intermediate between that of strains BEST, ST and wild-type DL-1 and also had an intermediate capacitance (Figure 6). There was a strong correlation $\left(r^{2}=0.998\right)$ between the capacitance and the cytochrome content of the different strains, suggesting that c-type cytochromes contribute to the biofilm capacitance.

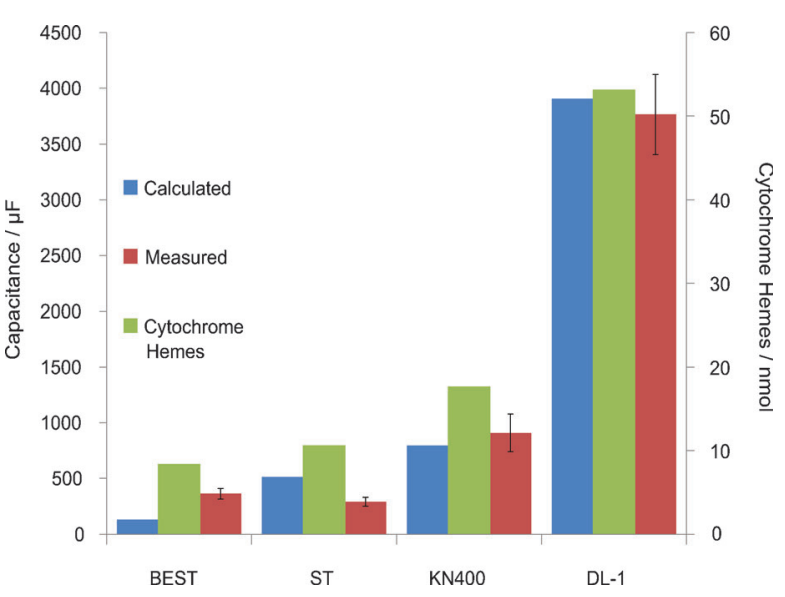

Figure 6. Comparison of capacitance of biofilms of various Geobacter sulfurreducens strains and their total cytochrome heme content. Blue: calculated capacitance; Green: cytochrome heme content; Red: Measured capacitance; Error bars: Standard deviation. Capacitance was evaluated with three-electrode electrochemical impedance spectroscopy.

If c-type cytochromes were responsible for the capacitance of the $G$. sulfurrreducens biofilms then it would be expected that this capacitance would be in the form of "pseudocapacitance" which is characteristic of fast and reversible redox reactions. ${ }^{[17]}$ Cyclic voltammetry of $G$. sulfurreducens biofilms displayed a set of anodic and cathodic peaks (Figure 7), consistent with pseuduocapacitance, ${ }^{[3,18]}$ whereas the voltammograms obtained for control electrodes in the absence of biofilm were rectangular (Figure 7), which is characteristic of pure electrostatic capacitance. ${ }^{[3,18]}$

The presence of pseudocapacitance in G. sulfurreducens biofilms was also evident from galvanostatic charge and discharge cycling (Figure 8). In this analysis, the biofilms clearly deviated from the ideal triangular shape expected for pure electrostatic double-layer capacitance. ${ }^{[3,18]}$ Such a deviation is typical of pseudocapacitive contributions. ${ }^{[3,17,18]}$ This result is consistent with the expected pseudocapacitive behavior of c-type cytochromes and further suggests that the large capacitance of the biofilm is due to the redox chemistry of c-type cytochromes. 


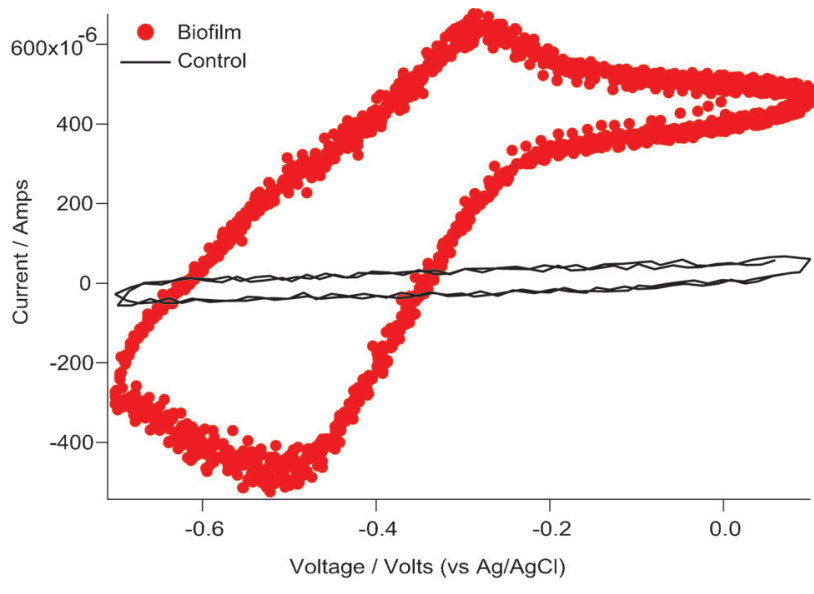

Figure 7. Representative cyclic voltammetry of Geobacter sulfurreducens biofilm (red curve) and the corresponding control electrodes (black curve) in the voltage range $-0.7 \mathrm{~V}$ to $0.1 \mathrm{~V}$ versus $\mathrm{Ag} / \mathrm{AgCl}$. Scan rate $100 \mathrm{mV} \mathrm{s}^{-1}$.

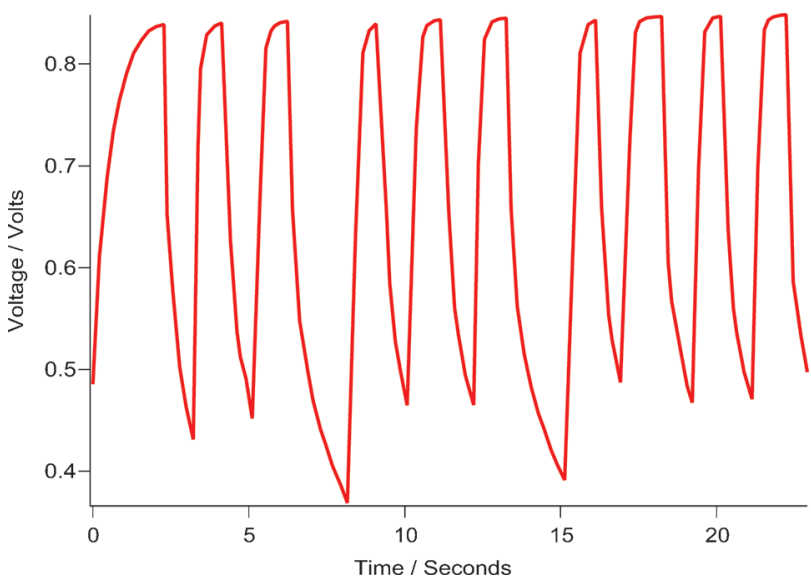

Figure 8. Typical galvanostatic charge and discharge profile of Geobacter sulfurreducens biofilm, at $1 \mathrm{~mA}$ applied current. electron), $F$ is the Faraday constant, $R$ is the molar gas constant and $T$ is the temperature. $\Delta E$ is the difference between the electrode potential $(E)$ and the formal potential $\left(E^{\prime}\right)$ of the biofilm. (See the Supporting Information for details.) The formal potential, measured using cyclic voltammetry, for each of the strains was: strain $\mathrm{DL}-1,-350 \mathrm{mV}$ vs $\mathrm{Ag} / \mathrm{AgCl}$; strain $\mathrm{KN} 400$, $-370 \mathrm{mV}$ vs $\mathrm{Ag} / \mathrm{AgCl}$; strain $\mathrm{ST},-330 \mathrm{mV}$ vs $\mathrm{Ag} / \mathrm{AgCl}$; strain BEST, $-390 \mathrm{mV}$ vs $\mathrm{Ag} / \mathrm{AgCl}$, which was comparable to the values previously reported in the literature. ${ }^{[19,20]} E$ is the electrode potential measured at open circuit condition which for the various strains was: strain $\mathrm{DL}-1,-450 \mathrm{mV}$ vs $\mathrm{Ag} / \mathrm{AgCl}$; strain $\mathrm{KN} 400,-483 \mathrm{mV}$ vs $\mathrm{Ag} / \mathrm{AgCl}$; strain $\mathrm{ST},-441 \mathrm{mV}$ vs $\mathrm{Ag}$ / $\mathrm{AgCl}$; strain BEST, $-530 \mathrm{mV}$ vs $\mathrm{Ag} / \mathrm{AgCl}$. Double-layer capacitance was neglected in the calculation of total capacitance, because its value is small for gold electrodes. ${ }^{[21]}$ There was good agreement between the pseudocapacitance calculated from heme abundance and the measured capacitance (Figure 6). These results further suggested that capacitance of the G. sulfurreducens biofilms could be attributed to the cytochromes.

\subsection{Unfolding the c-Type Cytochromes Diminishes the Biofilm Capacitance}

Addition of acetylmethionine, which denatures c-type cytochromes, ${ }^{[22]}$ diminished the capacitance of the biofilm as well as current production (Figure 9). In contrast, denaturing the cytochromes did not affect the conductivity of microbial nanowire networks. ${ }^{[12]}$ These experiments confirmed that the observed large enhancement of capacitance in biofilms is due to the charge storage ability of c-type cytochromes. The rate of inhibition of current production was faster than the loss of capacitance, which was attributed to the fact that flux of acetylmethionine into the biofilm which diminished the capacitance is rate limited by the diffusion, ${ }^{[23]}$ whereas the suppression in current is due to an instantaneous change in the redox potential of the $c$-type cytochromes. ${ }^{[22]}$

\subsection{Computed Capacitance Due to c-Type Cytochromes is Comparable with Measured Capacitance}

Pseudocapacitance due to redox-active species is defined as the derivative of charge acceptance and a change in potential, $^{[2]}$ and for c-type cytochromes, it can be calculated by Equation (1)::[1]

$C_{\varphi}=Q \frac{(F / R T) \exp (\Delta E \cdot F / R T)}{[1+\exp (\Delta E \cdot F / R T)]^{2}}$

where $Q$ is the total charge associated with the cytochrome hemes (each heme stores one

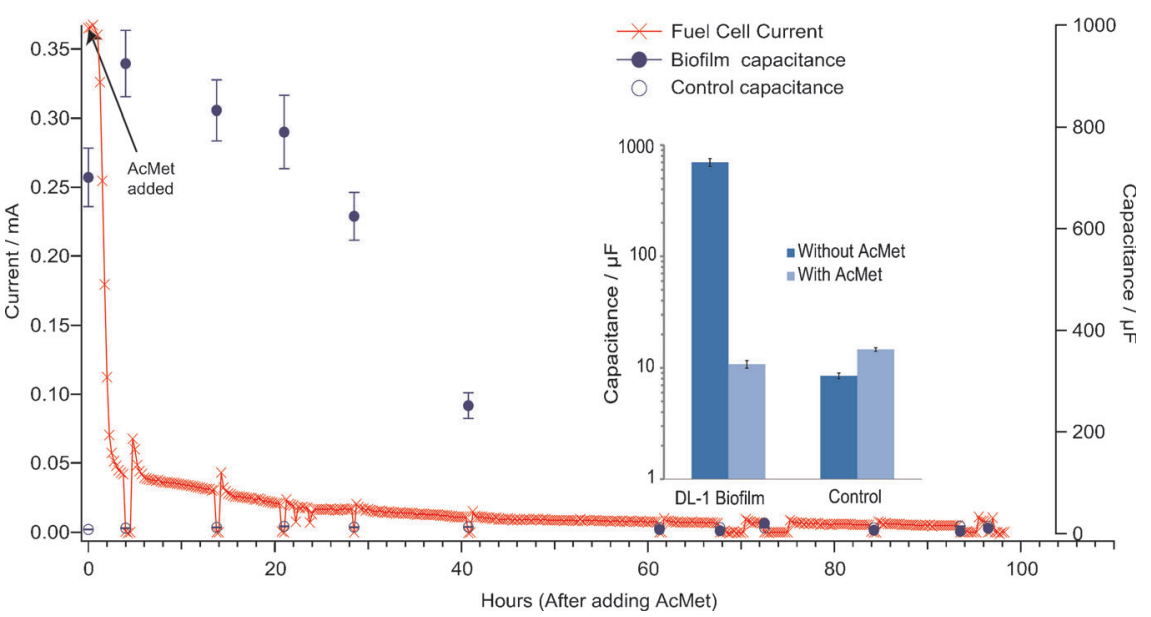

Figure 9. Capacitance of the biofilm (blue solid circles) and control electrodes (blue open circles) over hours after adding acetylmethionine (AcMet, $120 \mathrm{mM}$ ) at pH 7. Microbial current at the time of capacitance measurement is shown by red trace. Inset: Capacitance of biofilm and control before (dark blue bars) and after (light blue bars) adding AcMet for $100 \mathrm{~h}$. Error bars: Standard deviation. Capacitance was measured with two-electrode ac impedance spectroscopy. 


\subsection{G. sulfurreducens Biofilms Show Low Self-Discharge Behavior}

Self-discharge is a major limiting factor affecting the performance of supercapacitors. ${ }^{[17]}$ Self-discharge causes the opencircuit voltage of a charged capacitor to decrease over time. During self-discharge, the leakage current discharges the capacitor even in the absence of any external electrical current. The self-discharge is typically due to overcharging of the capacitor beyond the decomposition limit of the electrolyte or due to the result of internal redox reactions involving redoxactive molecules or impurities present in the electrolyte. ${ }^{[17]}$

When the self-discharge behavior of $G$. sulfurreducens biofilms was studied by monitoring the open-circuit potential, the voltage change was ca. $8 \mathrm{mV}$ after almost an hour (Figure 10).

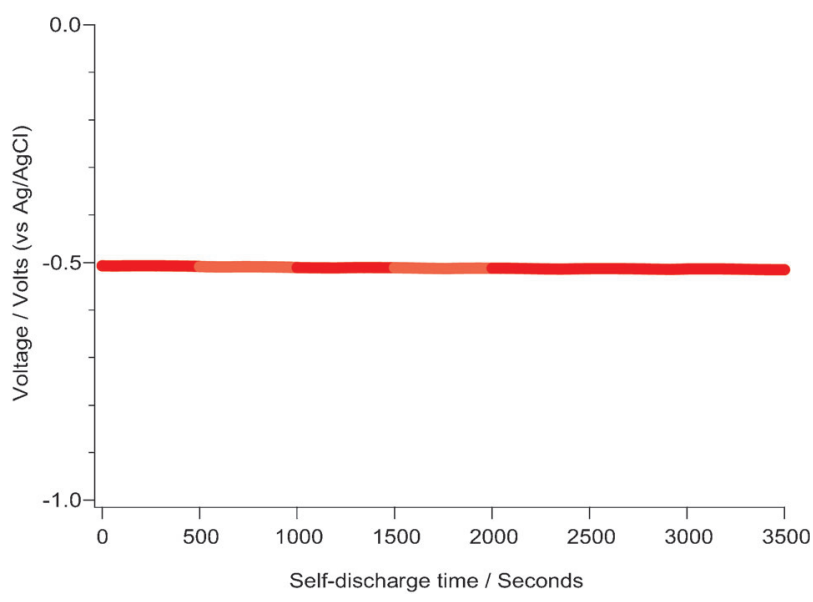

Figure 10. Self-discharge behavior of biofilm presented as the open-circuit potential measured against $\mathrm{Ag} / \mathrm{AgCl}$ versus time.

This corresponds to ca. $1.6 \%$ self -discharge in an hour with a rate of $2 \mu \mathrm{Vsec}^{-1}$ and the associated leakage current of ca. $0.3 \mathrm{mAg}^{-1}$ which is comparable to synthetic supercapacitors. ${ }^{[24-26]}$ These values indicate that $G$. sulfurreducens biofilms exhibit excellent self-discharge and leakage current behavior.

\subsection{Specific Capacitance of G. sulfurreducens Biofilms is Comparable with Synthetic Supercapacitors}

We used gold electrodes with dimensions of ca. 1 " $\times 1$ " $(2.54 \mathrm{~cm} \times 2.54 \mathrm{~cm})$, which corresponds to an effective surface area of $6.45 \mathrm{~cm}^{2}$. Therefore, the capacitance of $G$. sulfurreducens biofilms normalized per unit area is ca. $620 \mu \mathrm{F} \mathrm{cm}^{-2}$ at the open circuit potential. Treating cytochrome hemes as active materials, the computed specific capacitance of DL- 1 biofilm is $111 \mathrm{Fg}^{-1}$, which is comparable to synthetic supercapacitors. ${ }^{[1]}$

\section{Conclusions}

These results demonstrate that c-type cytochromes embedded in the conductive matrix of $G$. sulfurreducens biofilms impart a large capacitance to $G$. sulfurreducens biofilms that is compara- ble to synthetic supercapacitors. Multiple lines of evidence indicated that the c-type cytochromes are responsible for charge storage. Electrochemical impedance spectroscopy, cyclic voltammetry and the galvanostatic charge-discharge cycling, all demonstrated the pseudocapacitive properties of biofilms consistent with redox-active molecules being responsible for the capacitance. There was a strong correlation between cytochrome heme abundance and biofilm capacitance. Denaturing cytochromes removed capacitance, and the capacitance measured in biofilms with different cytochrome abundances compared well with the cytochrome-based pseudocapacitance calculated with a Nernstian, redox capacitance model. The biofilms showed good reversibility in charging and discharging processes, low leakage current and excellent self-discharge behavior. The superior electrochemical performance of the biofilm is related to its high abundance of cytochromes, its nanostructured network with metallic-like conductivity, and its porous architecture with hydrous nature. ${ }^{[2]}$

The finding that cytochrome-based biofilms can function as supercapacitors may open new possibilities for capacitor design. Biofilms can be grown from relatively inexpensive feedstocks and when alive, can self-repair and replicate, properties that distinguish them from abiological capacitors. Combining genetic engineering ${ }^{[4]}$ as well as novel electrode materials ${ }^{[27]}$ and electrolytes ${ }^{[3]}$ might significantly boost the performance of the supercapacitor devices using living microbes described herein.

\section{Experimental Section}

In order to directly measure the capacitance in vivo, biofilms of $G$. sulfurreducens strain DL-1 were grown in a microbial fuel cell by connecting a split-anode setup to a cathode, as described previously. ${ }^{[12]}$ Two gold electrodes $(2.54 \mathrm{~cm} \times 2.54 \mathrm{~cm})$ separated by a $50 \mu \mathrm{m}$ non-conductive gap were used as a split anode (Figure 1). Acetate $(10 \mathrm{mM})$ serves as an electron donor and the anode as the electron acceptor. Control electrodes were not connected to a cathode to prevent biofilm formation (Figure $1 \mathrm{~b}$ ). Capacitance was measured by temporarily disrupting the connection between the anode and cathode and applying ac voltage across the split electrodes (Figure $1 \mathrm{~b}$ ). All capacitance measurements were performed at the open circuit potential. At the open circuit, the anode potential becomes significantly negative (ca. $-500 \mathrm{mV}$ vs $\mathrm{Ag} / \mathrm{AgCl}$ ) and the bacteria cannot utilize the anode as an electron acceptor. Thus, the open circuit condition is equivalent to electron acceptor limitations faced by the bacteria in a subsurface environment where electron acceptors, like Fe ${ }^{\text {III }}$ oxides, are heterogeneously dispersed. Since the capacitance of the gap can limit the overall capacitance measured by two-electrode impedance spectroscopy, three-electrode electrochemical impedance spectroscopy was employed to better define the biofilm capacitance and an equivalent circuit model with a single time constant was used to extract capacitance

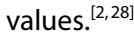

All bacterial strains were grown anaerobically as previously described. ${ }^{[12]}$ Cytochrome heme content was estimated by UV/Visible scanning of disrupted cells using UV2401-PC spectrophotometer (Shimadzu, MD, USA). The oxidized cytochromes had a distinct peak at $410 \mathrm{~nm}$ and the obtained absorbance value of that peak was used to calculate heme content by comparing to a standard 
curve made with oxidized bovine heart cytochrome $c$ (Sigma, MO, USA). For the specific capacitance calculation, the molecular weight of the heme $\left(616.5 \mathrm{~g} \mathrm{~mole}^{-1}\right)$ was used $\left(\mathrm{NIH}^{\prime}\right.$ s Molecular $\mathrm{Li}-$ braries Roadmap Initiative-Pubchem Compound).

All electrochemical experiments were performed using Solartron 1287 potentiostat/galvanostat at room temperature. Impedance spectroscopy was performed using a Solartron 1252/1287 impedance analyzer. Impedance was measured by sweeping frequency between $100 \mathrm{mHz}$ and $300 \mathrm{KHz}$ with $0.1 \mathrm{~V}$ amplitude ac voltage excitation. Data fitting was performed with ZView software (Scribner Inc.) which uses LEVM algorithm developed by J. Ross Macdonald. ${ }^{[28]}$ For three-electrode electrochemical impedance spectroscopy, a gold split electrode was used as a working electrode, the reference electrode was $\mathrm{Ag} / \mathrm{AgCl}, 3 \mathrm{M} \mathrm{KCl}(\mathrm{BAS}$, IN, USA) and the counter electrode was carbon cloth. ${ }^{[12]}$ For all impedance measurements, open circuit potential was monitored until it reached a constant value before over imposing ac signal. Cyclic voltammetry was performed in the voltage range $-0.7 \mathrm{~V}$ to $0.1 \mathrm{~V}$ vs $\mathrm{Ag} / \mathrm{AgCl}$ reference electrode at a scan rate of $100 \mathrm{mV} \mathrm{s}^{-1}$. The galvanostatic charge and discharge profiles were measured at the applied current of $1 \mathrm{~mA}^{[3]}$ The self-discharge behavior was observed by measuring the open-circuit potential of the electrode in the presence of biofilm versus $\mathrm{Ag} / \mathrm{AgCl}$ reference electrode. The data was collected and analyzed using CoreView software (Scribner Inc).

\section{Acknowledgements}

This work was supported by the Office of Naval Research (grant no. AN00014-10-1-0084), the office of Science (BER), US Department of Energy (award no. DE-SC0004114 and Cooperative Agreement no. DE-FC02-02ER63446) as well as the NSF Center for Hierarchical Manufacturing (grant no. CMMI-1025020). We thank Laura Francis for helpful discussions.

Keywords: bacteria $\cdot$ cytochromes $\cdot$ electrochemistry $\cdot$ redox chemistry $\cdot$ supercapacitors

[1] T. A. Skotheim, J.R. Reynolds, Handbook of Conducting Polymers, 2nd ed., CRC Press, Boca Raton, 2007.

[2] L. L. Zhang, X. S. Zhao, Chem. Soc. Rev. 2009, 38, 2520-2531.

[3] S. Roldán, C. Blanco, M. Granda, R. Menéndez, R. Santamaría, Angew. Chem. 2011, 123, 1737-1739; Angew. Chem. Int. Ed. 2011, 50, 16991701.

[4] Y. J. Lee, H. Yi, W. J. Kim, K. Kang, D. S. Yun, M. S. Strano, G. Ceder, A. M. Belcher, Science 2009, 324, 1051- 1055.

[5] D. R. Lovley, T. Ueki, T. Zhang, N. S. Malvankar, P. M. Shrestha, K. Flanagan, M. Aklujkar, J. E. Butler, L. Giloteaux, A. E. Rotaru, D. E. Holmes, A. E.
Franks, R. Orellana, C. Risso, K. P. Nevin, Adv. Microb. Physiol. 2011, 59 , $1-100$

[6] B. A. Methe, K. E. Nelson, J. A. Eisen, I. T. Paulsen, W. Nelson, J. F. Heidelberg, D. Wu, M. Wu, N. Ward, M. J. Beanan, R. J. Dodson, R. Madupu, L. M. Brinkac, S. C. Daugherty, R. T. DeBoy, A. S. Durkin, M. Gwinn, J. F. Kolonay, S. A. Sullivan, D. H. Haft, J. Selengut, T. M. Davidsen, N. Zafar, O. White, B. Tran, C. Romero, H. A. Forberger, J. Weidman, H. Khouri, T. V. Feldblyum, T. R. Utterback, S. E. Van Aken, D. R. Lovley, C. M. Fraser, Science 2003, 302, 1967-1969.

[7] A. Esteve-Núñnez, J. Sosnik, P. Visconti, D. R. Lovley, Environ. Microbiol. 2008, 10, 497-505

[8] D. R. Lovley, Geobiology 2008, 6, 225-231.

[9] J. Zhao, Y. Fang, T. D. Scheibe, D. R. Lovley, R. Mahadevan, J. Contam. Hydrol. 2010, 112, 30-44.

[10] Y. Liu, H. Kim, R. R. Franklin, D. R. Bond, ChemPhysChem 2011, 12, 2235 2241.

[11] D. Schrott Germán, B. P. Sebastian, R. Luciana, E.-N. Abraham, J. Pablo Busalmen, Electrochim. Acta 2011, 56, 10791 - 10795.

[12] N. S. Malvankar, M. Vargas, K. P. Nevin, A. E. Franks, C. Leang, B. C. Kim, K. Inoue, T. Mester, S. F. Covalla, J. P. Johnson, V. M. Rotello, M. T. Tuominen, D. R. Lovley, Nat. Nanotechnol. 2011, 6, 573-579.

[13] D. Herbert-Guillou, B. Tribollet, D. Festy, L. Kiéné, Electrochim. Acto 1999, 45, 1067-1075.

[14] J. W. Voordeckers, B. C. Kim, M. Izallalen, D. R. Lovley, Appl. Environ. Microbiol. 2010, 76, $2371-2375$.

[15] T. Mehta, M. V. Coppi, S. E. Childers, D. R. Lovley, Appl. Environ. Microbiol. 2005, 71, 8634-8641.

[16] H. Yi, K. P. Nevin, B. C. Kim, A. E. Franks, A. Klimes, L. M. Tender, D. R. Lovley, Biosens. Bioelectron. 2009, 24, 3498-3503.

[17] B. E. Conway, Electrochemical Supercapacitors: Scientific Fundamentals and Technological Applications, Kluwer/Plenum, New York, 1999.

[18] S. Roldán, Z. González, C. Blanco, M. Granda, R. Menéndez, R. Santamaría, Electrochim. Acta 2011, 56, $3401-3405$.

[19] H. Richter, K. P. Nevin, H. Jia, D. A. Lowy, D. R. Lovley, L. M. Tender, Energy Environ. Sci. 2009, 2, 506-516.

[20] S. M. Strycharz, A. P. Malanoski, R. M. Snider, H. Yi, D. R. Lovley, L. M. Tender, Energy Environ. Sci. 2011, 4, 896-913.

[21] E. Katz, I. Willner, Electroanalysis 2003, 15, 913-947.

[22] R. Nakamura, F. Kai, A. Okamoto, G. J. Newton, K. Hashimoto, Angew. Chem. 2009, 121, 516-519; Angew. Chem. Int. Ed. 2009, 48, 508-511.

[23] S. J. Hagen, J. Hofrichter, A. Szabo, W. A. Eaton, Proc. Natl. Acad. Sci. USA 1996, 93, 11615-11617.

[24] E. Frackowiak, S. Delpeux, K. Jurewicz, K. Szostak, D. Cazorla-Amoros, F. Beguin, Chem. Phys. Lett. 2002, 361, 35-41.

[25] K. Fic, G. Lota, E. Frackowiak, Electrochim. Acta 2010, 55, 7484-7488.

[26] A. B. Fuertes, G. Lota, T. A. Centeno, E. Frackowiak, Electrochim. Acta 2005, 50, 2799-2805.

[27] Y. Zhu, S. Murali, M. D. Stoller, K. J. Ganesh, W. Cai, P. J. Ferreira, A. Pirkle, R. M. Wallace, K. A. Cychosz, M. Thommes, D. Su, E. A. Stach, R. S. Ruoff, Science 2011, 332, 1537-1541.

[28] E. Barsoukov, J. R. Macdonald, Impedance Spectroscopy: Theory, Experiment and Applications, 2nd ed., Wiley, Hoboken, 2005.

Received: October 28, 2011

Published online on January 17,201 